\title{
5. Tomografía computada de alta resolución en el diagnóstico de la fibrosis pulmonar idiopática
}

\author{
JUAN CARLOS DÍAZ P.*, TAMARA PALAVECINO B.** y GONZALO PEREIRA R.**
}

High resolution computed tomography in the diagnosis of idiopathic pulmonary fibrosis

Usual interstitial pneumonia pattern (UIP) in high-resolution computed tomography (HRCT) has a high degree of correlation with the histological pattern which makes it possible to obviate in these cases the need for lung biopsy. The accuracy of a UIP diagnosis in HRCT is based on the detection of specific signs of fibrosis such as honeycomb and reticular densities with traction bronchiolectasis. The update of the 2018 Clinical Practice Guidelines of the ATS / ERS / JRS / ALAT of Idiopathic Pulmonary Fibrosis (IPF) and the Fleischner Society White Paper proposed a new way of classifying the tomographic patterns taking into account the aspects previously considered. Although the presence of honeycomb remains the main finding to characterize the UIP pattern, the distribution of interstitial reticulate and the presence of traction bronchiolectasis, in the specific clinical context, may be sufficient to make the diagnosis of idiopathic pulmonary fibrosis.

Key words: Idiopathic Pulmonary Fibrosis; Tomography, X-Ray computed; Thorax; Traction bronchiolectasis.

\section{Resumen}

El patrón de neumonía intersticial usual (NIU) en la tomografía computada de alta resolución (TCAR) tiene un alto grado de correlación con el patrón histológico lo que permite obviar en dichos casos la necesidad de realizar biopsia pulmonar. La exactitud del diagnóstico de NIU en TCAR se basa en la detección de signos especificos de fibrosis como el panal y las densidades reticulares con bronquiolectasias por tracción. La actualización de las guías 2018 de práctica clínica de la ATS/ERS/JRS/ ALAT de Fibrosis Pulmonar Idiopática (FPI) y la declaración de consenso de la Sociedad Fleischner proponen una nueva forma de clasificar los patrones tomográficos tomando en cuenta los aspectos antes considerados. Si bien la presencia de panal sigue siendo el hallazgo principal para caracterizar el patrón NIU, la distribución del reticulado intersticial y la presencia de bronquiolectasias por tracción en el contexto clínico especifico puede ser suficiente para realizar el diagnóstico de fibrosis pulmonar idiopática.

Palabras clave: Fibrosis pulmonar idiopática; Tomografía Computada de tórax; Bronquioloectasias por tracción.

La fibrosis pulmonar idiopática tiene como sustrato histológico el patrón de neumonía intersticial usual (NIU). Cuando se identifican los hallazgos característicos, la tomografía computada de alta resolución (TCAR) tiene un alto grado de correlación con este patrón histológico, obviando en esos casos la necesidad de realizar biopsia ${ }^{1}$.

\section{Consideraciones técnicas}

Para un óptimo rendimiento diagnóstico de la tomografía computada, es esencial obtener imágenes de alta calidad ${ }^{2}$. Esto significa adquirir las imágenes en inspiración profunda, con cortes finos $(<2 \mathrm{~mm})$ y reconstrucción de alta resolu-

* Hospital Clínico Universidad de Chile. Profesor asociado Universidad de Chile. Santiago, Chile.

** Hospital Clínico Universidad de Chile. Profesor asistente Universidad de Chile. Santiago, Chile. 
Tabla 1. Resumen técnica tomografía computada de alta resolución ${ }^{1}$

\begin{tabular}{|ll|}
\hline Recomendado & \\
Espesor de corte & $0,5-1,5 \mathrm{~mm}$ \\
Algoritmo de reconstrucción & De alta frecuencia espacial \\
$\mathrm{KV}(\mathrm{p})$ & 120 \\
$\mathrm{~mA}$ & menos de $250 ; \mathrm{mA}$ efectivo de 100 o menos \\
Tiempo de rotación & lo más corto posible $(0,3-0,5 \mathrm{~s})$ \\
Pitch (multidetector) & $1-1,5$ \\
Nivel de inspiración & inspiración profunda \\
Posición & Supina, agregando cortes en posición prono en pacientes con densidades en zonas \\
& gravitatorio-dependientes (basales periféricas posteriores) \\
Tipo de adquisición & Volumétrico \\
Reconstrucción & Transaxial de todo el tórax \\
Ventanas & Nivel: $600-700$ HU, amplitud: $1.000-1.500$ HU. Combinaciones sugeridas $700 / 1.000$, \\
Visualización de imágenes & E00/1.500. \\
Opcional & Baja do trabajo (óptimo) \\
Reducción mA & Para pacientes con deterioro respiratorio agudo para excluir tromboembolia pulmonar \\
Contraste ev & Niveles inferiores (800-900) para detección de enfisema. Para enfermedad pleuropar- \\
Ventana & quimatosa se recomienda $600 / 2000$ HU \\
Imágenes en espiración & Secuencial en 3 o más niveles \\
\hline
\end{tabular}

$\mathrm{HRCT}=$ high resolution computed tomography, $H U=$ unidades Hounsfield .

ción espacial. La inspiración inadecuada puede aumentar la atenuación pulmonar y producir falsas imágenes de vidrio esmerilado o retículo fino. Para una mejor delimitación de la extensión de la enfermedad y de la distribución de las alteraciones, se prefiere la adquisición volumétrica a la adquisición seriada. Se recomiendan imágenes en decúbito prono cuando hay densidades periféricas en zonas dependientes en las imágenes en decúbito supino. Pueden ser de utilidad los cortes en espiración para confirmar atrapamiento aéreo, particularmente cuando se sospecha diagnósticos alternativos como Neumonitis por Hipersensibilidad (NH) y enfermedad del tejido conectivo (ETC). Los detalles técnicos recomendados para realizar el examen se detallan en la Tabla 1.

\section{Definición de patrón neumonia intersticial usual}

La exactitud del diagnóstico de NIU en TCAR se basa en la detección de signos de fibrosis con una distribución particular ${ }^{3}$. Los signos más específicos de fibrosis son el panal y las densidades reticulares con bronquiolectasias por tracción.

\section{Panal}

El panal corresponde a la agrupación de espacios quísticos de contenido aéreo, de diámetros relativamente similares, en el orden de 3 a 10 $\mathrm{mm}$ (ocasionalmente pueden alcanzar hasta 25 $\mathrm{mm}$ de diámetro). Sus características principales se muestran en la Figura 1.

La sobreposición de vidrio esmerilado en zonas de enfisema, puede generar imágenes de difícil interpretación al acentuar los bordes de los

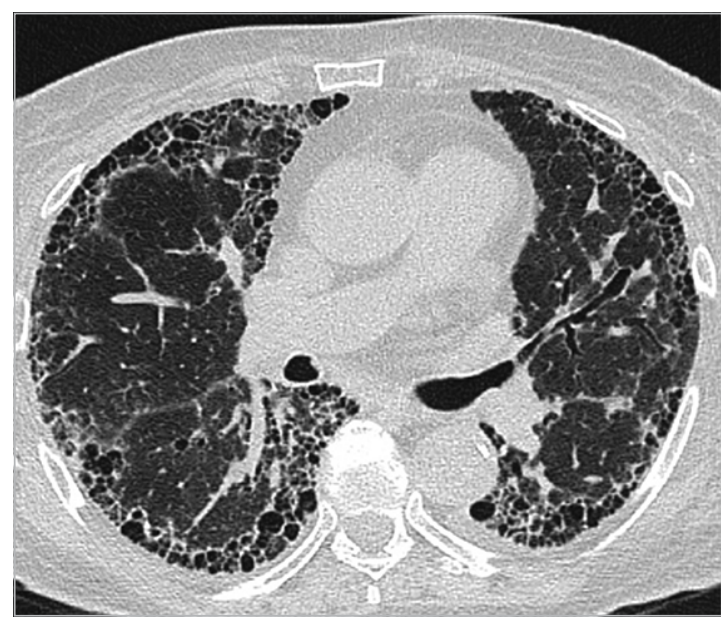

Figura 1. Corte axial de TC bajo el nivel de la carina, muestra formaciones quísticas con contenido aéreo agrupadas y adosadas a la superficie pleural, con paredes compartidas, bien definidas y gruesas. El tamaño de los quistes es relativamente homogéneo y no sobrepasan los $10 \mathrm{~mm}$. 

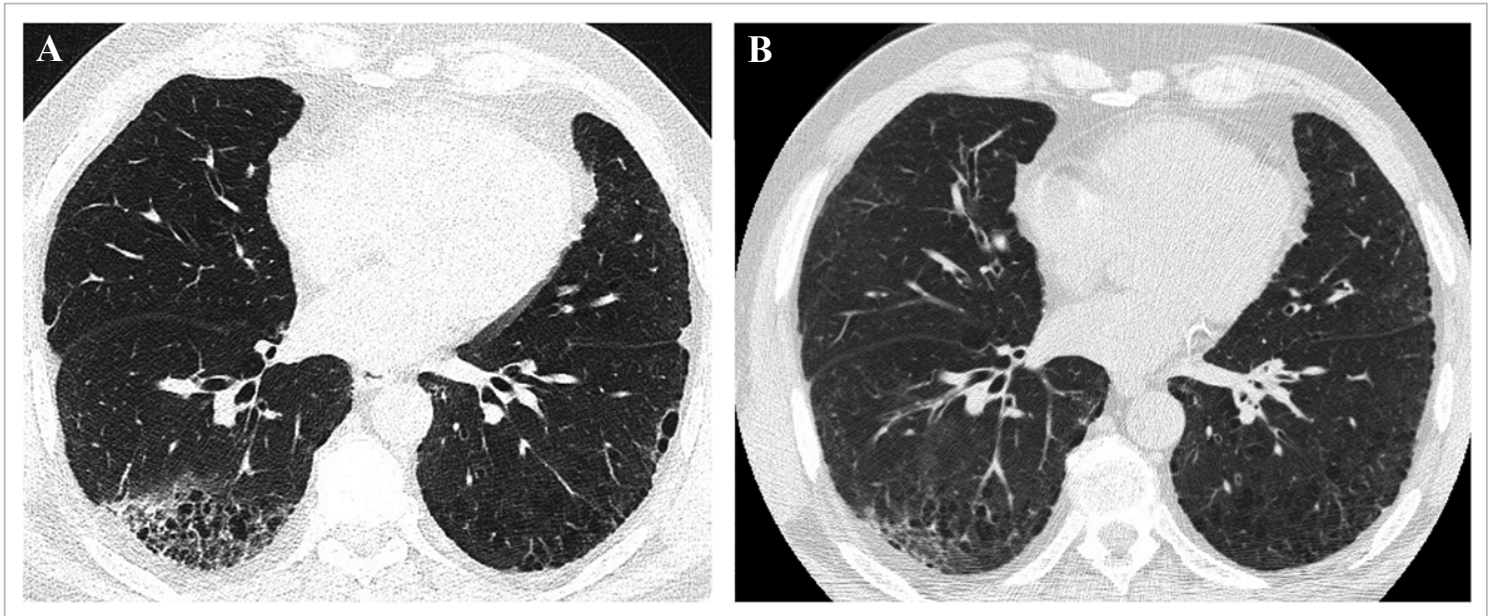

Figura 2. A. Corte axial de TC muestra espacios enfisematosos con opacidades en vidrio esmerilado en el LID, acentuando las estructuras intersticiales y simulando retículo o panal. A diferencia del panal, no todos los quistes tienen paredes bien definidas, no todos comparten sus paredes y no están adosados a la superficie pleural. B. Posterior a la resolución parcial del vidrio esmerilado, se comprueba que las imágenes quísticas corresponden a espacios enfisematosos y no a panal.

espacios enfisematosos y simular panal ${ }^{4}$. Es importante, entonces, revisar que las características de panal mencionadas estén presentes, es decir, que las formaciones quísticas estén adosadas a la superficie pleural y que compartan sus paredes. En ocasiones, sólo el control puede resolver la duda (Figura 2).

También son de difícil diagnóstico aquellos casos en que el panal se dispone en una hilera de quistes pudiendo ser confundido con enfisema paraseptal ${ }^{5}$. Para evitar el sobrediagnóstico, se considera panal a esta presentación, sólo cuando los quistes se ubican en los lóbulos inferiores, son de tamaño relativamente uniforme, de paredes gruesas, compartidas y no se identifica áreas de enfisema en el parénquima adyacente (Figura 3).

En aquellos pacientes en que coexiste enfisema paraseptal en los tercios superiores y medios, puede ser imposible establecer el límite entre ambos procesos.

\section{Patrón reticular intralobulillar}

Se caracteriza por líneas finas que se entrecruzan formando una red. En los pacientes con NIU, las líneas son de distintos grosores y los espacios entre ellas son irregulares, en contraste con el retículo de la neumonía intersticial inespecífica, en general de grosor más homogéneo y espacios regulares (Figura 4).

\section{Bronquiolectasias por tracción}

El panal de abejas constituye la etapa final de una neumonía intersticial. En la NIU con frecuencia el panal se asocia a opacidades en vidrio esmerilado y retículo intralobulillar, que histológicamente corresponden a una mezcla de dilatación de la vía aérea terminal y fibrosis

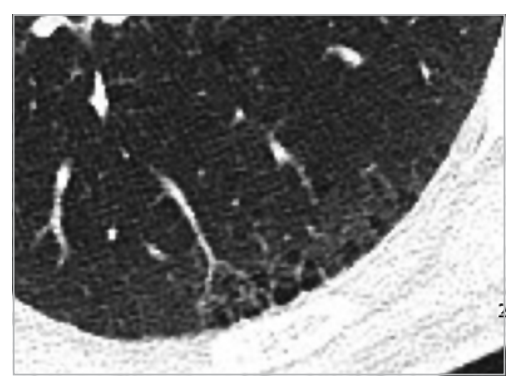

Figura 3. Imágenes de contenido aéreo subpleurales en una hilera. Sus paredes son imperceptibles y están separadas por parénquima pulmonar. Esto corresponde a enfisema paraseptal.

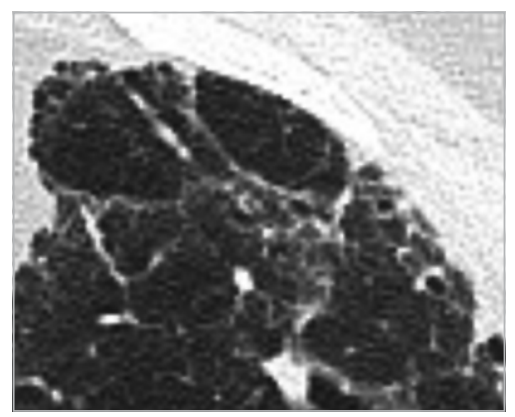

Figura 4. TC muestra foco de retículo intralobulillar con líneas de distinto grosor y con espacios irregulares en el lóbulo superior izquierdo. 
periacinar. Si bien estas imágenes pueden indicar fibrosis, son inespecíficas. Con el tiempo, el retículo intralobulillar aumenta y gradualmente aparecen bronquiolectasias por tracción, que corresponden a dilatación de bronquios y bronquíolos dentro del lobulillo por la fibrosis retráctil del parénquima circundante ${ }^{6}$ (Figura 5). Para algunos autores, la dilatación se produce por proliferación anormal del epitelio bronquiolar, como parte de un espectro de remodelación bronquiolar. El panal correspondería a la etapa final de este proceso, de modo que la separación conceptual entre bronquiolectasia y panal sería un error ${ }^{7}$. Cualquiera sea el mecanismo de formación, las bronquiolectasias constituyen un signo altamente específico de fibrosis pulmonar, incluso en presencia de enfisema. Dependiendo de la orientación del corte también pueden presentarse como formaciones quísticas con contenido aéreo difíciles de distinguir del panal (Figura 5). Por lo demás, estudios de correlación anatomo/ radiológica, muestran que estas dos alteraciones a menudo coexisten ${ }^{8}$. Por este motivo nos parece que la distinción no es relevante desde el punto de vista diagnóstico pues histológicamente ambas corresponden a fibrosis y su presencia y extensión tiene igual pronóstico que el panal ${ }^{9}$.

\section{Distribución}

La localización de las alteraciones, predominantemente en la periferia de los sectores basales y posteriores de los pulmones, es la característica más específica de la NIU junto con los signos de fibrosis. En estos sitios del pulmón es donde se produce el mayor estrés mecánico en los movimientos respiratorios, pudiendo desencadenar así la formación de desgarros microscópicos que pueden dar lugar a pequeños eventos de cicatrización y, eventualmente, a la formación de panal de abeja ${ }^{10}$. Para otros autores, el colapso alveolar secundario al daño de sus paredes sería el principal evento fisiopatológico responsable de la progresión de la NIU/FPI. De acuerdo a esto, los segmentos basales posteriores de los lóbulos inferiores debieran ser los más gravemente implicados, ya que es aquí donde están los alvéolos más pequeños en las posiciones vertical y supina $\mathrm{y}$, por lo tanto, los más propensos a colapsar ${ }^{11}$.

En las etapas iniciales, el compromiso periférico se distribuye en forma heterogénea a lo largo de la pleura, observándose con frecuencia áreas de respeto. No es infrecuente encontrar algún grado de asimetría en el compromiso pulmonar. El panal subpleural en las bases, casi siempre se acompaña de densidades reticulares en las zonas pulmonares superiores, produciéndose un gradiente con mayor compromiso de las bases.

En resumen, el patrón de NIU en la TCAR de tórax puede describirse de la siguiente manera (Tabla 2): "hallazgos de fibrosis subpleural en las bases pulmonares, con una distribución heterogénea y al menos retículo en las regiones superiores". Como distribución heterogénea se entenderá la visualización de todos los estados de la enfermedad en un mismo corte de la TC, alternando zonas normales, con otras de retículo y panal (Figura 6). Cuando los elementos de fibrosis tienen una distribución categóricamente subpleural y heterogénea, nos parece irrelevante intentar diferenciar entre panal y bronquiolecta-

\section{Tabla 2 . Patrón de neumonia intersticial usual}

\section{Eje axial: Predominio subpleural}

Eje cráneo caudal: Predominio basal o sin predominio en lóbulos superiores

Panal y bronquiectasias por tracción con o sin retículo

Compromiso heterogéneo (en etapas iniciales)
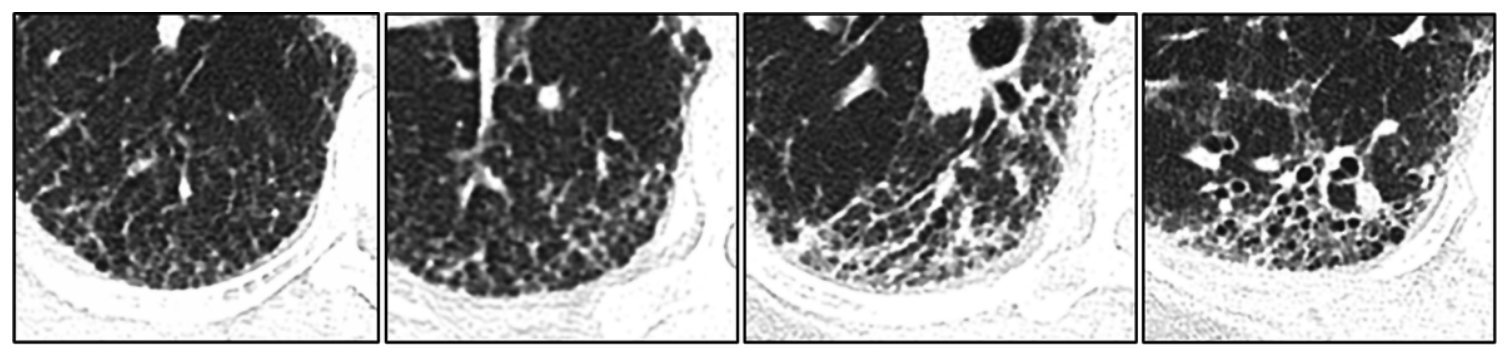

Figura 5. De izquierda a derecha se observa progresión del retículo con aparición creciente de estructuras arrosariadas con contenido aéreo, correspondientes a dilatación de bronquiolos intralobulillares (bronquiolectasias por tracción). En la imagen del extremo derecho se observan varias formaciones quísticas agrupadas que corresponden a bronquiolos dilatados en corte axial. Puede confundirse con panal, pero a diferencia del panal, en muchas se puede ver parénquima pulmonar entre ellas (no comparten pared). 


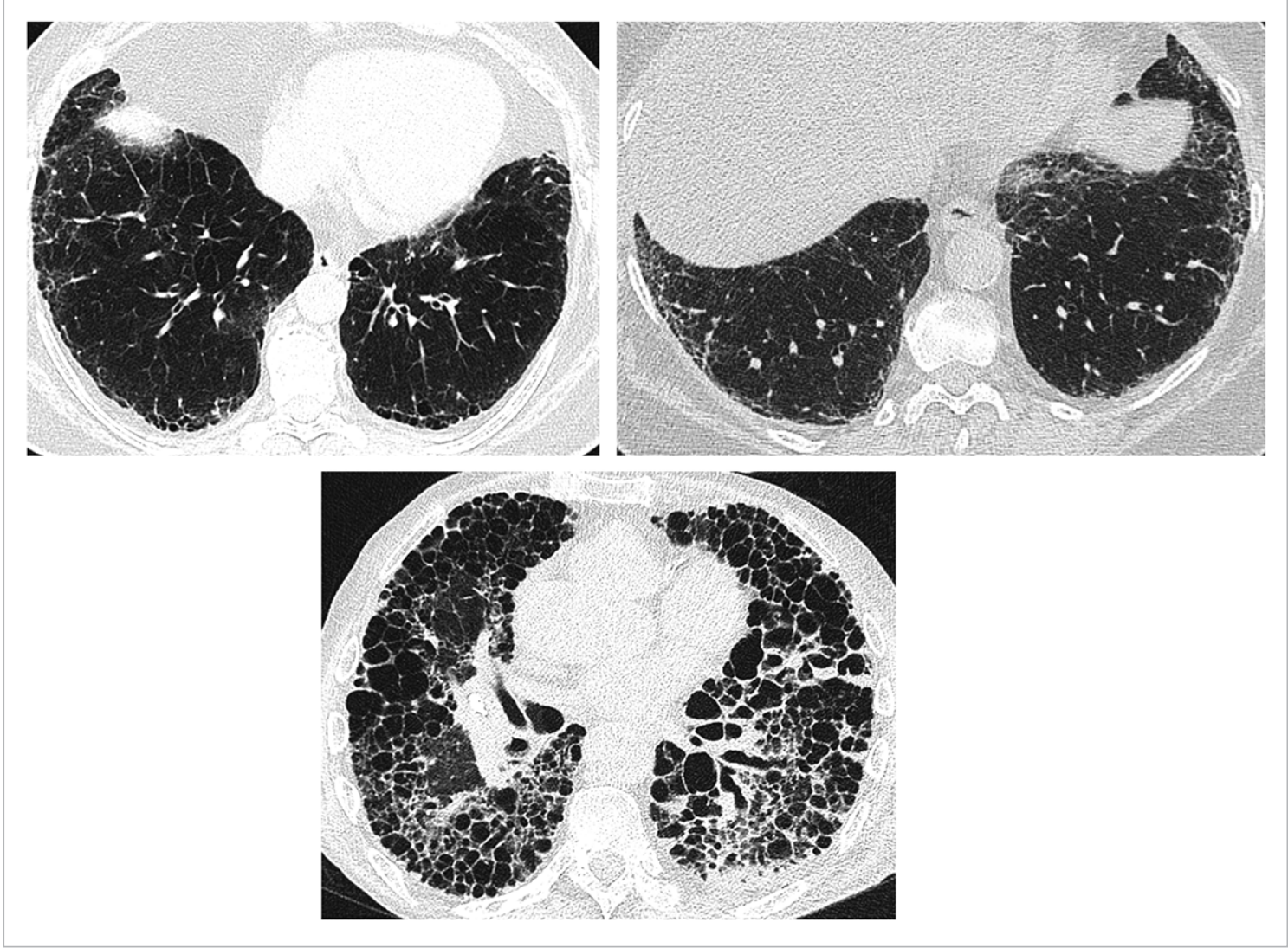

Figura 6. En los cortes axiales de TC de la fila superior se observa signos de fibrosis (con panal categórico a izquierda y dudoso a derecha), subpleural y de distribucion heterogénea. En la fila inferior se observa un estadío avanzado con panal exuberante, en que el compromiso se ha extendido hacia la región central y en forma más homogénea.

sias por las razones antes expuestas. Sin embargo, en etapas avanzadas, estas características de distribución tienden a desaparecer observándose un compromiso más homogéneo y con mayor extensión hacia el centro del pulmón (Figura 6). En esos casos sí resulta relevante diferenciar el panal de las bronquiolectasias por tracción, pues el compromiso homogéneo asociado solo a bronquiolectasias por tracción es altamente sugerente de Neumonia intersticial no específica (NINE) fibrosante, particularmente si presenta distribución peribroncovascular y con respeto subpleural (Figura 7).

Otros hallazgos posibles y que no descartan un patrón de NIU:

1) Distribución asimétrica.

2) Mosaico en áreas de fibrosis.

3) Compromiso peribroncovascular cuando corresponde a extensión del compromiso subpleural.
4) Vidrio esmerilado extenso asociado a un patrón UIP. En estos casos se debe considerar: exacerbación, infección por germen oportunista, insuficiencia cardiaca.

Hallazgos que sugieren un diagnóstico alternativo:

- Anormalidades pleurales:

- placas pleurales, calcificaciones pleurales: asbestosis.

- Derrame pleural: ETC.

- Respeto subpleural: NINE.

- Mosaico en áreas sin fibrosis: Neumonitis por hipersensibilidad (NHS).

- Fibrosis peribroncovascular exclusiva o predominante: NHS-ETC.

- Fibrosis de lóbulos superiores exclusiva o predominante: silicosis, sarcoidosis.

- Compromiso homogéneo: NINE.

Dos recientes publicaciones sobre el diagnóstico de FPI, la actualización de las Guías de 


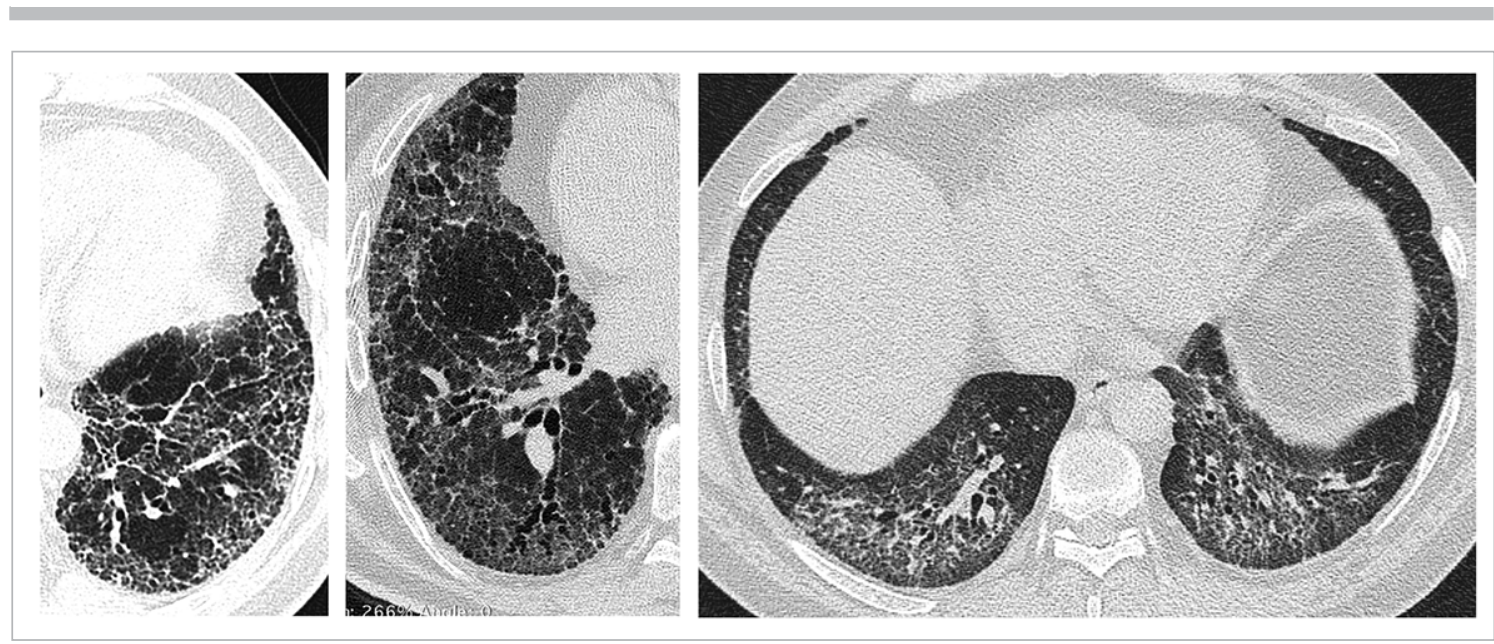

Figura 7. Cortes axiales de TC que muestran compromiso de distribución más continua, sin zonas normales de interrupción, y sin exclusividad de la zona subpleural. La fibrosis se expresa solo por bronquiolectasias por tracción sin evidencias de panal. En el corte de la fila inferior se observa además marcada distribución peribroncovascular, con respeto subpleural, elementos que hacen pensar en diagnóstico alternativo a NIU.

Práctica clínica de la ATS/ERS/JRS/ALAT ${ }^{12}$ de FPI y la declaración de Consenso de la Sociedad Fleischner ${ }^{13}$, proponen una nueva forma de clasificar los patrones tomográficos tomando en cuenta los aspectos antes considerados. Ambos documentos eliminan el patrón de "posible NIU" de la clasificación anterior y establecen las siguientes cuatro categorías: patrón NIU, patrón NIU probable, patrón indeterminado y patrones de diagnóstico alternativo. Uno de los aportes de esta nueva clasificación es que abre la posibilidad de hacer diagnóstico de FPI sin necesidad de biopsia quirúrgica en los pacientes con patrón de probable NIU en un contexto clínico adecuado. Si bien ambas coinciden en establecer la presencia de panal como clave para el patrón NIU definitivo, también coinciden en que el acuerdo interobservador para identificación de éste es solo moderado. Por esta razón, nos parece relevante enfatizar que la distribución de los cambios fibróticos es también importante en la identificación del patrón tomográfico, de modo que en casos de fibrosis con distribución típica de NIU (subpleural basal y heterogénea), en contexto clínico adecuado, recomendamos categorizarlas como patrón NIU definitivo aun cuando exista duda respecto a si los cambios fibróticos corresponden a panal o bronquiolectasias por tracción.

\section{Conclusión}

La TCAR tiene un papel central en el algoritmo de estudio de la FPI, aportando los patrones definitivo y probable de NIU, que permiten obviar la biopsia quirúrgica en contextos clínicos adecuados.

\section{Bibliografía}

1.- SUNDARAM B, GROSS BH, MARTÍNEZ FJ, OH E, MÜLLER NL, SCHIPPER M, et al. Accuracy of high-resolution $\mathrm{CT}$ in the diagnosis of diffuse lung disease: effect of predominance and distribution of findings. AJR Am J Roentgenol 2008; 191: 1032-9.

2.- MAYO JR. CT evaluation of diffuse infiltrative lung disease: dose considerations and optimal technique. J Thorac Imaging 2009; 24: 252-9.

3.- HUNNINGHAKE GW, LYNCH DA, GALVIN JR, GROSS BH, MÜLLER N, SCHWARTZ DA, et al. Radiologic findings are strongly associated with a pathologic diagnosis of usual interstitial pneumonia. Chest 2003; 124: 1215-23.

4.- AKIRA M, INOUE Y, KITAICHI M, YAMAMOTO S, ARAI T, TOYOKAWA K. Usual interstitial pneumonia and nonspecific interstitial pneumonia with and without concurrent emphysema: thin-section CT findings. Radiology 2009; 251: 271-9.

5.- WATADANI T, SAKAI F, JOHKOH T, NOMA S, AKIRA M, FUJIMOTO K, et al. Interobserver variability in the CT assessment of honeycombing in the lungs. Radiology 2013; 266: 936-44.

6.- WEBB WR, MÜLLER NL, NAIDICH DP. Highresolution $\mathrm{CT}$ of the lung 4th Ed. Lippincot Williams and Wilkins. Philadelphia, USA, 2009.

7.- PICIUCCHI S, TOMASSETTI S, RAVAGLIA C, GURIOLI C, GURIOLI C, DUBINI A, et al. From 
"traction bronchiectasis" to honeycombing in idiopathic pulmonary fibrosis: a spectrum of bronchiolar remodeling also in radiology? BMC Pulm Med 2016; $16: 87$.

8.- STAATS P, KLIGERMAN S, TODD N, TAVORA F, XU L, BURKE A. A comparative study of honeycombing on high resolution computed tomography with histologic lung remodeling in explants with usual interstitial pneumonia. Pathol Res Pract 2015; 211: 55-61.

9.- EDEY AJ, DEVARAJ AA, BARKER RP, NICHOLSON AG, WELLS AU, HANSELL DM. Fibrotic idiopathic interstitial pneumonias: HRCT findings that predict mortality. Eur Radiol 2011; 21: 1586-93.

10.- CARLONI A, POLETTI V, FERMO L, BELLOMO N, CHILOSI M. Heterogeneous distribution of mechanical stress in human lung: a mathematical approach to evaluate abnormal remodeling in IPF. J Theor Biol 2013; 332: 136-40.

11.- GALVIN JR, FRAZIER AA, FRANKS TJ. Collaborative radiologic and histopathologic assessment of fibrotic lung disease. Radiology 2010; 255: 692-706.

12.- RAGHU G, REMY-JARDIN M, MYERS JL, RICHELDI L, RYERSON CJ, LEDERER DJ, et al. Diagnosis of idiopathic pulmonary fibrosis: an official ATS/ERS/JRS/ALAT clinical practice guideline. Am J Respir Crit Care Med 2018; 198: e44-e68.

13.- LYNCH DA, SVERZELLATI N, TRAVIS WD, BROWN KK, COLBY TV, GALVIN JG, et al. Diagnostic criteria for idiopathic pulmonary fibrosis: a Fleischner Society White Paper. Lancet Respir Med 2018; 6: 138-53. 